\title{
Estimating agonist efficiency from concentration-response curves
}

\author{
Dinesh Indurthi ${ }^{1}$ and Anthony Auerbach ${ }^{1}$ \\ ${ }^{1}$ University at Buffalo - The State University of New York
}

May 5, 2020

\begin{abstract}
Background and Purpose: Agonists turn on receptors because they have higher affinity for active versus resting conformations of their target sites. Agonist efficiency (distinct from efficacy) is the fraction of binding energy applied to the receptor's activating conformational change and depends only on the resting/active equilibrium dissociation constant ratio. Our goal was to estimate agonist efficiency from concentration-response curves (CRCs). Experimental Approach: Adult skeletal muscle acetylcholine receptors (AChRs) were expressed in HEK cells and CRCs were compiled from single-channel currents. The efficiencies of 13 agonists were estimated from the midpoints and maxima of the CRCs by using equations that pertain to a cyclic activation scheme. Key Results: Agonist efficiency was greater for small- compared to large-volume agonists, $52 \pm 2 \%$ $(\mathrm{n}=9)$ for 70 A3 versus $40 \pm 5 \%(\mathrm{n}=4)$ for 101 A3. The smoking-cessation drugs varenicline and cytisine belong to the lower efficiency group. An examination of AChRs having a binding site mutation showed that ?Y190A was the only one of 22 that affected the efficiency of ACh, switching it from the more- to the less-efficient group. IF agonist efficiency is known, EC50 can be estimated from the maximum response and the unliganded gating equilibrium constant can be calculated from the CRC parameters. Conclusion and Implications: We hypothesize that larger agonists have lower efficiencies because they limit the extent of binding-pocket contraction upon activation, and that efficiency values may be modal. Knowledge of agonist efficiency simplifies and extends CRC analysis.
\end{abstract}

\section{Introduction}

Nicotinic AChRs are ligand-gated ion channels that couple an increase in agonist binding energy to an increase in gating equilibrium constant. These receptors switch globally between resting and active conformations ('gating') that bind agonists weakly (with low affinity) and have a C(losed) channel, or strongly (with high affinity) and have an $\mathrm{O}$ (pen) channel (Fig. 1) (Monod, Wyman \& Changeux, 1965). Constitutive AChR activation is uphill energetically, so without a bound agonist(s) the probability of being in the $\mathrm{O}$ conformation $\left(\mathrm{P}_{\mathrm{O}}\right)$ is vanishingly small (Jackson, 1986; Nayak, Purohit \& Auerbach, 2012; Purohit \& Auerbach, 2009). However, when a resting receptor with a bound agonist(s) begins spontaneously to undergo the channelopening transition, the newfound, favorable binding energy serves to increase $\mathrm{P}_{\mathrm{O}}$ above the basal level. This increase can be dramatic. At adult human neuromuscular synapses, 2 bound neurotransmitter molecules increase transiently $\mathrm{P}_{\mathrm{O}}$ from 0.0000005 to 0.95 (Auerbach, 2012; Karlin, 1967).

Agonists are differentiated by two features of their CRCs, the concentration that produces a half-maximal response ( $\mathrm{EC}_{50}$, related to 'affinity') and the maximum response ( $\mathrm{P}_{\mathrm{O}}{ }^{\max }$, related to 'efficacy') (Colquhoun, 1998). Recently, a third distinguishing attribute, 'efficiency', was proposed (Nayak, Vij, Bruhova, Shandilya \& Auerbach, 2020). Agonist efficiency is defined as the fraction of ligand binding energy that is converted into the mechanical energy of the gating conformational change. As described below, agonist affinity, efficacy and efficiency all are functions of the equilibrium dissociation constants for binding to the resting state $\left(\mathrm{K}_{\mathrm{dC}}\right)$ and to the active state $\left(\mathrm{K}_{\mathrm{dO}}\right)$.

Previously, $\mathrm{K}_{\mathrm{dC}}$ and $\mathrm{K}_{\mathrm{dO}}$ for agonists related structurally to either ACh or the frog toxin epibatidine were measured at individual human AChR neurotransmitter binding sites (Nayak \& Auerbach, 2017). Kinetic 
analyses of single-channel currents showed that the average efficiency of agonists related to ACh was greater than for agonists related to epibatidine. Investigations of corresponding AChR structures showed that binding energy is correlated linearly and inversely with the distance between a key nitrogen atom in the ligand and the center of a binding cavity $\left(\mathrm{d}_{\mathrm{x}}\right)$ that contracts upon receptor activation (Tripathy, Zheng \& Auerbach, 2019). Further, these studies showed that the bound ligand is more centered in the active versus resting pocket, and that agonist efficiency can be estimated from the relative change in $\mathrm{d}_{\mathrm{x}}$ upon activation.

Below, we calculate agonist efficiency from $\mathrm{EC}_{50}$ and $\mathrm{P}_{\mathrm{O}}{ }^{\max }$ values for agonists of adult-type mouse endplate AChRs. We report efficiencies for 16 agonists, as well as for $\mathrm{ACh}$ in receptors having point mutations at the binding sites. The efficiencies fall into 2 groups, with larger-volume ligands being $\sim 15 \%$ less efficient than smaller-volume ligands. Knowledge of agonist efficiency simplifies and extends CRC analysis.

\section{Methods}

Expression, electrophysiology, analysis. Human embryonic kidney (HEK) 293 cells were maintained in Dulbecco's minimal essential medium supplemented with $10 \%$ fetal bovine serum and $1 \%$ penicillinstreptomycin, $\mathrm{pH}$ 7.4. AChRs were expressed by transient transfection of mouse $\alpha, \beta, \delta$, and $\varepsilon$ subunits in the ratio 2:1:1:1 (Trans IT ${ }^{\circledR} 293$ transfection reagent; Mirus Bio, Madison, WI). Electrophysiological experiments started $\sim 48 \mathrm{hrs}$ post-transfection. No animals were used in this study.

Our goal was to calculate agonist efficiencies from equilibrium dissociation constants extracted from CRCs, by using equations derived from Scheme 1 (Fig. 1). Whole-cell currents contain events that are not included in this scheme, for instance from desensitization or from modal activity. In order to match the 'response' $\left(\mathrm{P}_{\mathrm{O}}\right)$ with Scheme 1 as closely as possible, we constructed pseudo-macroscopic CRCs from single-channel currents, as follows.

Single-channel currents were recorded in the cell-attached patch configuration at $23^{\circ} \mathrm{C}$. The bath solution was (in mM): $142 \mathrm{KCl}, 5.4 \mathrm{NaCl}, 1.8 \mathrm{CaCl}_{2}, 1.7 \mathrm{MgCl}_{2}, 10 \mathrm{HEPES} / \mathrm{KOH}$ (pH 7.4). Patch pipettes were fabricated from borosilicate glass and fire polished to a resistance of $\sim 10 \mathrm{M}$ when filled with the pipette solution that was Dulbecco's phosphate-buffered saline (in mM): $137 \mathrm{NaCl}, 0.9 \mathrm{CaCl}_{2}, 2.7 \mathrm{KCl}, 1.5 \mathrm{KH}_{2} \mathrm{PO}_{4}, 0.5 \mathrm{MgCl}_{2}$, and $8.1 \mathrm{Na}_{2} \mathrm{HPO}_{4}(\mathrm{pH} 7.3 / \mathrm{NaOH})$. Currents were recorded using a PC505 amplifier (Warner instruments, Hamden, CT), low-pass filtered at $20 \mathrm{kHz}$ and digitized at a sampling frequency of $50 \mathrm{kHz}$ using a data acquisition board (SCB-68, National instruments, Austin, TX). Agonists were added to the pipette solution at the desired concentration.

When the diliganded opening rate constant $\left(\mathrm{A}_{2} \mathrm{C}-\mathrm{A}_{2} \mathrm{O}\right.$ in Fig. 1) is sufficiently large, $\mathrm{AChR}$ openings occur in clusters (Sakmann, Patlak \& Neher, 1980). Shut intervals within clusters represent mainly agonist-binding and receptor-gating events (Fig. 1, Scheme 2) whereas shut intervals between clusters represent mainly longlived desensitized events. We selected for analysis clusters that appeared by eye to arise from a homogeneous $\mathrm{P}_{\mathrm{O}}$ population, and limited the analysis to intra-cluster events in order to remove sojourns in long-lived desensitized states from the responses.

Because of the high extracellular $\left[\mathrm{K}^{+}\right]$, the cell membrane potential $\left(\mathrm{V}_{\mathrm{m}}\right)$ was $0 \mathrm{mV}$. The $\mathrm{AChR}$ agonists we examined also are channel-blockers. To both generate measurable currents and reduce the effect of channel block on $\mathrm{P}_{\mathrm{O}}$, the membrane was depolarized to $+70 \mathrm{mV}$ by holding the pipette at $-70 \mathrm{mV}$. This effectively eliminated agonist binding to the blocking site in the transmembrane domain but did not affect agonist binding to the neurotransmitter sites in the extracellular domain.

Analyses of the (outward) currents were performed by using QUB software (Nicolai \& Sachs, 2013). A cluster was defined as a group of openings flanked by shut intervals [?]7 ms. Currents within clusters were idealized into noise-free intervals by using the segmental k-means algorithm (SKM) after digitally low-pass filtering the data at $10 \mathrm{kHz}$ (Qin, 2004). Distributions of idealized interval durations were fitted by multiple exponential components using a maximum interval likelihood algorithm (MIL)(Qin, Auerbach \& Sachs, 1997). Starting with a kinetic model having one shut and one open state (C-O), additional shut states were connected to $\mathrm{O}$ until the $\log$ likelihood no longer improved by $>10$ units. Usually only 1 extra shut state was necessary. 
$\mathrm{P}_{\mathrm{O}}$ was calculated from the time constant of the predominant component of the shut time distribution $\left(\tau_{\mathrm{s}}\right)$ and the time constant of the open time distribution $\left(\tau_{\mathrm{o}}\right): \tau_{\mathrm{o}} /\left(\tau_{\mathrm{s}}+\tau_{\mathrm{o}}\right)$ (Purohit \& Grosman, 2006). With this approach, short-duration shut intervals within clusters arising from sojourns in a short-lived desensitized state were eliminated from the accounting (Elenes \& Auerbach, 2002). The CRCs reflect absolute $\mathrm{P}_{\mathrm{O}}$ values and were not normalized to a maximum value.

Estimating binding constants from CRC parameters . The midpoint, maximum and slope of the $\mathrm{CRC}\left(\mathrm{EC}_{50}\right.$, $\mathrm{P}_{\mathrm{O}}{ }^{\max }$, and $\mathrm{n}$ ) were estimated by fitting by an empirical equation,

$$
\mathrm{P}_{\mathrm{O}}=\mathrm{P}_{\mathrm{O}}{ }^{\max } /\left(1+\left(\mathrm{EC}_{50} /[\text { agonist }]\right)^{\mathrm{n}}\right) \text { Eq. } 1
$$

We used 3 equations to calculate $\mathrm{K}_{\mathrm{dC}}$ and $\mathrm{K}_{\mathrm{dO}}$ from $\mathrm{EC}_{50}$ and $\mathrm{P}_{\mathrm{O}}{ }^{\max }$. From microscopic reversibility and Scheme 1,

$$
\frac{E_{2}}{E_{0}}=\left(\frac{K_{\mathrm{dC}}}{K_{\mathrm{dO}}}\right)^{2} \text { Eq. } 2
$$

$\mathrm{E}_{2}$ is the diliganded gating equilibrium constant and $\mathrm{E}_{0}$ is the unliganded (intrinsic) gating equilibrium constant. The exponent reflects that in adult-type AChRs there are 2 approximately equivalent and independent neurotransmitter binding sites (Nayak \& Auerbach, 2017).

Constitutive and mono-liganded activation are infrequent so in wild-type AChRs the main pathway connecting $\mathrm{C}$ to $\mathrm{A}_{2} \mathrm{O}$ (unliganded-resting to diliganded-active) is Scheme 2 (Fig. 1). In terms of equilibrium constants, the CRC parameters for Scheme 2 are:

$$
\mathrm{EC}_{50}=\frac{K_{\mathrm{dC}} \sqrt{E_{2}+2}}{E_{2}+1} \text { Eq. } 3 P_{O}^{\max }=\frac{1}{1+\frac{1}{E_{2}}} \text { Eq. } 4
$$

The procedure for calculating $\mathrm{K}_{\mathrm{dC}}$ and $\mathrm{K}_{\mathrm{dO}}$ from the CRC parameters was as follows. We calculated $\mathrm{E}_{2}$ from $\mathrm{P}_{\mathrm{O}}$ max using Eq. 4, then calculated $\mathrm{K}_{\mathrm{dC}}$ from $\mathrm{E}_{2}$ and $\mathrm{EC}_{50}$ using Eq. 3, then calculated $\mathrm{K}_{\mathrm{dO}}$ from $\mathrm{E}_{2}$ and $\mathrm{K}_{\mathrm{dC}}$ using Eq. 2 and a known value of $\mathrm{E}_{0}$ (see below).

A k-means cluster analysis algorithm (Matlab) was used to define groups regarding agonist efficiency and volume.

Background mutations . Depolarization to $\mathrm{V}_{\mathrm{m}}=+70 \mathrm{mV}$ (to reduce channel block by the agonist) has the undesired consequence of shortening $\tau_{0}$ to make current detection and idealization more difficult. To compensate, we added the background mutation $\varepsilon \mathrm{S} 450 \mathrm{~W}$ (in the M4 transmembrane segment of the $\varepsilon$ subunit) that has the equal-but-opposite effect on gating as does depolarization by $+140 \mathrm{mV}$ but does not alter agonist binding (Jadey, Purohit, Bruhova, Gregg \& Auerbach, 2011). With this mutation, $\tau_{\mathrm{O}}$ and the unliganded gating equilibrium constant $\mathrm{E}_{0}$ at $+70 \mathrm{mV}$ were the same as in wild-, adult-type AChRs at -70 $\mathrm{mV} . \mathrm{E}_{0}$ at $-100 \mathrm{mV}$ is $7.4 \times 10^{-7}$ and is reduced e-fold with a $60 \mathrm{mV}$ depolarization (Nayak, Purohit \& Auerbach, 2012), so we estimate that $\mathrm{E}_{\mathrm{o}}=4.5 \times 10^{-7}$ at $\mathrm{V}_{\mathrm{m}}=+70 \mathrm{mV}$ with $\varepsilon \mathrm{S} 450 \mathrm{~W}$.

Clusters of open-current intervals were poorly defined with the low-efficacy agonist varenicline. To increase the diliganded opening rate constant and generate higher $\mathrm{P}_{\mathrm{O}}$ clusters we added two background mutations in the $\varepsilon$ subunit, $\varepsilon \mathrm{L} 269 \mathrm{~F}$ (in the M2 helix) and $\varepsilon \mathrm{E} 181 \mathrm{~W}$ (in strand $\beta 9$ ) without $\varepsilon S 450 \mathrm{~W}$. Together, these two substitutions increase the unliganded gating equilibrium constant by 1084 -fold $\left(\mathrm{E}_{0}{ }^{\text {mut }}=4.9 \times 10^{-4}\right)$ without affecting agonist binding (Jha, Purohit \& Auerbach, 2009; Purohit, Gupta, Jadey \& Auerbach, 2013).

Agonists. Agonist structures are shown in Figs. 2 and 3. The agonist head-group volumes were calculated using Chimera (Pettersen et al., 2004). Cytisine and varenicline were from Sigma ${ }^{\circledR}$ lifesciences (St. Louis, $\mathrm{MO}$ ). Epibatidine was obtained from Tocris Biosciences (Briston, UK). The sources for other agonists is in a previous publication (Bruhova \& Auerbach, 2017).

\section{Results}

Agonist efficiency 
Vertebrate neuromuscular AChRs have 2 neurotransmitter binding sites located in the extracellular domain at $\alpha$-d and $\alpha$-e subunit interfaces. Three previous experimental results make it possible to calculate $\mathrm{K}_{\mathrm{dC}}$ and $\mathrm{K}_{\mathrm{dO}}$ from the CRC parameters $\mathrm{EC}_{50}$ and $\mathrm{P}_{\mathrm{O}}{ }^{\max }$. First, in adult-type AChRs the two binding sites have approximately the same affinity for ACh and other agonists, so only single values of the constants needed to be estimated for each ligand. Second, Scheme 1 has been proved experimentally to describe receptor activation, so $\mathrm{K}_{\mathrm{dC}}$ could be calculated using Eqs. 3 and 4. Third, $\mathrm{E}_{\mathrm{o}}$ and its voltage dependence are known, so $\mathrm{K}_{\mathrm{dO}}$ could be calculated using Eq. 2.

Some authors use 'efficacy' and 'efficiency' interchangeably, but here we use these words to describe different agonist attributes. In our use, efficacy relates to the high-concentration asymptote of the CRC (the maximum response, set by equilibrium constant $\mathrm{E}_{2}$ ) relative to the zero-concentration asymptote (the constitutive response, set by equilibrium constant $\mathrm{E}_{0}$ ). As shown by Eq. 2, efficacy depends on the $\mathrm{K}_{\mathrm{dC}} / \mathrm{K}_{\mathrm{dO}}$ ratio. The $\log$ of an equilibrium dissociation constant is inversely proportional to binding energy, so agonist efficacy depends on thedifference in binding energies, $\mathrm{O}$ minus $\mathrm{C}$.

An energy value pertains to the free energy difference between end states. For instance, in Scheme 1, the diliganded gating energy (that is proportional to $\log \mathrm{E}_{2}$ ) is equal to the free energy difference between states $\mathrm{A}_{2} \mathrm{O}$ and $\mathrm{A}_{2} \mathrm{C}$. These energy differences are not influenced by short-lived intermediate states, for example flip (Lape, Colquhoun \& Sivilotti, 2008), prime (Mukhtasimova, Lee, Wang \& Sine, 2009) and phi (Purohit, Gupta, Jadey \& Auerbach, 2013) in gating. In our use, 'efficacy' is determined by everything that happens in the step $\mathrm{A}_{2} \mathrm{C}[?] \mathrm{A}_{2} \mathrm{O}$, as given by the overall equilibrium constant $\mathrm{E}_{2}$.

In contrast, 'efficiency' ( $\eta$ ' eta) is the useful output energy of a machine divided by the total input energy (Schroeder, 2000). In a receptor, the useful output energy is related to the maximum response relative to the baseline (that is, efficacy) and the total input energy is that for agonist binding to the active state. Hence (Nayak, Vij, Bruhova, Shandilya \& Auerbach, 2020),

$$
\eta=1-\log \mathrm{K}_{\mathrm{dC}} / \log \mathrm{K}_{\mathrm{dO}} \text {. Eq. } 5
$$

Agonist efficiency depends on the ratio of binding energies, C versus O. In AChRs the distance $\mathrm{d}_{\mathrm{x}}$ is correlated inversely with binding energy, so agonist efficiency can be estimated from structures as the ratio of $\mathrm{d}_{\mathrm{x}}$ values, $\mathrm{O}$ versus $\mathrm{C}$.

To highlight the distinction between efficacy and efficiency, consider the actions of carbamylcholine (CCh) and epiboxidine (Ebx) at the human $\alpha$-d site (Nayak \& Auerbach, 2017). These two ligands produce nearly the same gating equilibrium constant and, hence, have approximately the same efficacy. However, Ebx has a 84-fold higher resting affinity. Hence, CCh is the more-efficient ligand because a greater fraction of its (weaker) binding energy is used to generate the same gating response. This difference in efficiency is also apparent in structures. At the $\alpha$-d binding site, $\mathrm{d}_{\mathrm{x}}$ is smaller in $\mathrm{O}$ versus $\mathrm{C}$ by ${ }^{\sim} 1.9$-fold with $\mathrm{CCh}$ but only by 1.6 fold with Ebx (Tripathy, Zheng \& Auerbach, 2019).

CRCs for 7 agonists of adult-type mouse AChRs have been published (Jadey \& Auerbach, 2012; Jadey, Purohit \& Auerbach, 2013). We used Eq. 1 to estimate $\mathrm{EC}_{50}$ and $\mathrm{P}_{\mathrm{O}}{ }^{\max }$ from these, and Eqns. 2-5 to calculate agonist efficiencies from the fitted CRC parameters (Fig. 2 and Table 1). Although these 7 agonists span a wide range with regard to both $\mathrm{EC}_{50}(43 \mu \mathrm{M}$ to $6.7 \mathrm{mM})$ and $\mathrm{P}_{\mathrm{O}}{ }^{\max }(0.26$ to 0.96$)$, they all have approximately the same efficiency, $53+2 \%$ (mean + s.d). This efficiency value is similar to those calculated from equilibrium dissociation constants estimated by kinetic modeling. It is also approximately the same as the average efficiency of ACh, CCh, TMA and choline at individual $\alpha$-d and $\alpha$-e human neurotransmitter binding sites. Overall, the efficiencies estimated from CRCs are the approximately same i) as estimated from modeling, ii) for all 7 ligands, iii) at the two adult-type sites and iv) in mouse and human AChRs.

Next, we measured efficiencies from CRCs for 6 agonists that were not studied previously by CRC analysis (Fig. 3, Table 1). Choline (Cho) has 2 methylenes between its quaternary nitrogen and hydroxyl (OH) group, whereas 3OH-BTMA and 4OH-PTMA have 3 and 4. Cho is a low-affinity, low-efficacy agonist (Purohit \& Grosman, 2006) that has an efficiency at the human $\alpha$-e site of $52 \%$ (Nayak, Vij, Bruhova, Shandilya \& 
Auerbach, 2020). Simulations of structures suggest that an H-bond between the $\mathrm{OH}$ group of Cho and the backbone carbonyl of $\alpha \mathrm{W} 149$ serves to position the charged quaternary ammonium (QA) group away from the center of the binding cavity, thereby reducing the binding energy (Bruhova, Gregg \& Auerbach, 2013; Tripathy, Zheng \& Auerbach, 2019). Nonetheless, the opening transition reduces $\mathrm{d}_{\mathrm{x}}$ approximately by half with Cho, as it does with the higher-affinity, similar-efficiency agonists ACh, CCh and TMA.

$\mathrm{P}_{\mathrm{O}}{ }^{\text {max }}$ values for Cho, 3OH-BTMA and 4OH-PTMA are 0.05, 0.17 and 0.34 , respectively (Table 1 ). Nonetheless, the efficiencies of 3OH-BTMA and 4OH-PTMA calculated from the CRC parameters are same at 51\%, similar to Cho (50\%). These 3 structurally-related agonists have widely different affinities and efficacies but approximately the same efficiency as for the ligands shown in Fig. 2.

We also investigated CRCs for ligands related structurally to Ebx (Fig. 3, Table 1). The efficiency estimates for Epi and Ebx were similar to the values at the human $\alpha$-d binding site. Analyses of CRCs for two drugs that are used for smoking cessation, cytisine and varenicline, gave efficiencies of $42 \%$ and $35 \%$.

Efficiencies have been measured for 16 agonists. These were either estimated from midpoints and maxima of CRCs (Table 1) or calculated from published equilibrium dissociation constants (Nayak, Vij, Bruhova, Shandilya \& Auerbach, 2020). Fig. 4 shows these values are clustered, with one group $(n=10)$ having $\eta=52+$ $2 \%$ and another $(\mathrm{n}=6)$ having $\eta=40+5 \%$. Fig. 4 also shows that the volume of the 'head' group of the higher-efficiency agonists is smaller $\left(70+8 \mathrm{~A}^{3}\right)$ than that of the lower-efficiency agonists $\left(101+11.2 \mathrm{~A}^{3}\right)$.

To probe for residues that might influence efficiency, we estimated $\eta^{\mathrm{ACh}}$ from published equilibrium dissociation constants measured by kinetic modeling of single-channel currents in mouse adult-type AChRs having a mutation of a binding site residue (Purohit, Bruhova, Gupta \& Auerbach, 2014). Fig. 5 shows that for 21 of 22 mutants, $\eta^{\mathrm{ACh}}$ was $52+4 \%$ or the same as in the WT. The one exception was $\alpha$ Y190A for which $\eta^{\mathrm{ACh}}$ was $35 \%$.

Putting efficiency to use.

In this section we show that knowledge of agonist efficiency can simplify and extend CRC analysis. A group of agonists having the same efficiency means that for all members, the resting and active equilibrium dissociation constants are correlated exponentially. From Eq. 5,

$$
\mathrm{K}_{\mathrm{dO}}=\mathrm{K}_{\mathrm{dC}}{ }^{1 /(1-)} \text { Eq. } 6
$$

This relationship simplifies CRC analysis because there are fewer efficiency values than there are agonists, and because if $\eta$ is known one of the equilibrium dissociation constants can be calculated from the other. It may be possible to known an agonist's efficiency a priori, either by assuming it is the same as for a structurally-related ligand or by calculating it from the $\mathrm{d}_{\mathrm{x}}$ ratio in binding site structures.

Knowledge of $\eta$ (and the agonist-independent constant $\mathrm{E}_{0}$ ) allows the estimation of $\mathrm{EC}_{50}$ from the response at a single [agonist]. Combining Eqs. 2 and 6 and rearranging,

$$
\log \mathrm{E}_{2}=[2 \eta /(\eta-1)] \log \mathrm{K}_{\mathrm{dC}}+\log \mathrm{E}_{0} \mathrm{Eq} .7
$$

$\mathrm{E}_{2}$ can be calculated directly from $\mathrm{P}_{\mathrm{O}}{ }^{\max }$ (Eq. 4). Hence, given $\eta$ and $\mathrm{E}_{0}$ it is possible to solve Eq. 7 for $\mathrm{K}_{\mathrm{dC}}$, then solve Eq. 3 for $\mathrm{EC}_{50}$. Fig. $6 \mathrm{~A}$ shows that $\mathrm{EC}_{50}$ values so-calculated (assuming $\mathrm{E}_{\mathrm{o}}$ is $4.5 \times 10^{-7}$ and $\eta$ is either $52 \%$ or $40 \%$ ) match those obtained by fitting experimental CRCs. Further, CRCs calculated from just the high-concentration asymptote describe approximately responses at all [agonist] (Fig. 6B). If agonist efficiency is known, an entire $\mathrm{CRC}$ can be estimated from $\mathrm{P}_{\mathrm{O}}$ max .

Diliganded gating equilibrium constants have been measured experimentally for several different AChR agonists (Bruhova, Gregg \& Auerbach, 2013). Table 2 shows the corresponding, calculated $\mathrm{EC}_{50}$ values.

Knowledge of $\eta$ also allows the estimation of $\mathrm{E}_{0}$ from a single CRC. $\mathrm{E}_{0}$ is an important, ligand-independent constant that sets the basal level from which agonists increase $\mathrm{P}_{\mathrm{O}}$, but it can be difficult to measure. The procedure to estimate $\mathrm{E}_{0}$ from a CRC is first to solve for $\mathrm{E}_{2}$ and $\mathrm{K}_{\mathrm{dC}}$ from $\mathrm{P}_{\mathrm{O}}{ }^{\text {max }}$ and $\mathrm{EC}_{50}$ as described above, and then solve for $\mathrm{E}_{0}$ using Eq. 7. Fig. 7 shows $\mathrm{E}_{0}$ values calculated from the CRC parameters using 
an $\eta$ value of either $52 \%$ or $40 \%$. The mean result, $7.8 \times 10^{-7}$, is within a factor of 2 of the correct value, 4.5 $\mathrm{x} 10^{-7}$ (see Methods). If agonist efficiency is known, an approximate value of the intrinsic gating constant can be estimated from a single CRC.

If there are $\mathrm{E}_{2}$ and $\mathrm{K}_{\mathrm{dC}}$ estimates for multiple agonists, efficiency can be estimated by using an efficiency plot (Nayak, Vij, Bruhova, Shandilya \& Auerbach, 2020).

\section{Discussion and Conclusions}

Agonists are distinguished by 3 attributes that are functions of the binding energies to low- and high-affinity conformations of their target site. 'Affinity' is proportional to the binding energy itself $\left(\log \mathrm{K}_{\mathrm{dC}}\right.$ or $\left.\log \mathrm{K}_{\mathrm{dO}}\right)$, 'efficacy' is proportional to the binding energy difference $\left(\log \mathrm{K}_{\mathrm{dO}}-\log \mathrm{K}_{\mathrm{dC}}\right)$ and 'efficiency' depends only on the binding energy ratio $\left(\log \mathrm{K}_{\mathrm{dC}} / \log \mathrm{K}_{\mathrm{dO}}\right)$. The two equilibrium dissociation constants can be estimated in any number of different ways, for instance by kinetic modeling of single-channel currents or by a ligand binding assay. Here, we have shown that the unliganded gating equilibrium constant and the equilibrium dissociation constants (and, hence, agonist efficiency) can be estimated from a single dose-response curve.

The agonists related structurally to ACh have an average efficiency of $52 \%$ (Fig. 4). This value is approximately the same in human/mouse and at $\alpha$-d/a-e binding sites. The absence of a correlation between efficiency values estimated from CRCs versus by kinetic modeling suggests that the narrow range of efficiencies (50-55\%; Table 1) can be attributed to measurement errors rather than to actual, ligand-specific differences.

The ACh-occupied neurotransmitter binding cavity in equilibrated homology models of $\alpha$-d and $\alpha$-e sites has a volume of $\sim 120 \AA^{3}$ in the resting state and $\sim 90 \AA^{3}$ in the active state (Tripathy, Zheng \& Auerbach, 2019). The average volume of the head group for the $\sim 52 \%$ efficiency agonists is $\sim 70 \AA^{3}$. Hence, it is likely that all of these ligands fit comfortably in both the $\mathrm{C}$ and $\mathrm{O}$ conformations of the binding pocket.

Another group of agonists related structurally to Epi has a lower efficiency than for the ACh group. For these, we are less certain if the more-substantial range in efficiency, from $35 \%$ for varenicline to $46 \%$ for Ebx, can be attributed to measurement errors or to real differences between ligands. Epi has the same efficiency in whole receptors as at the isolated $\alpha$-d site, but the efficiency of Ebx is somewhat higher in whole receptors. It is possible that the efficiency of Ebx is modestly greater at $\alpha$-e compared to $\alpha$-d.

All of the low-efficiency agonists had a bridge moiety and, hence a larger head-group volume that on average was $101 \AA^{3}$. Hence, these ligands likely fit comfortably within the $\mathrm{C}$ conformation of the pocket but not within the $\mathrm{O}$ conformation. The inverse relationship between efficiency and head-group volume leads us to speculate that a large head group limits pocket contraction upon activation, to limit energy coupling to the rest of the extracellular domain, to limit agonist efficiency.

$\eta$ is a quantitative index of the extent of energy coupling between binding and gating and may thus shed light on the structural link(s) between these two fundamental processes. The mutation $\alpha \mathrm{Y} 190 \mathrm{~A}$ is the only binding site mutation we have discovered so far that alters ACh efficiency, reducing it from $50 \%$ to $35 \%$. Linear free energy analyses of mutant AChRs suggest that within the opening transition, the binding pocket and the extracellular domain rearrange sequentially (in that order) (Gupta \& Auerbach, 2011), and structural analyses suggest that both of these regions contract and rotate anticlockwise in the opening process (Sauguet et al., 2014) . The reduction in efficiency caused by $\alpha$ Y190A supports the suggestion that energy flows out of the binding pocket, in part, through this residue (Mukhtasimova, Free \& Sine, 2005). F and W substitutions at $\alpha \mathrm{Y} 190$ do not reduce efficiency so the energy transfer may involve the aromatic ring rather than the hydroxyl group. A more-extensive map of the effects of mutations on agonist efficiency might define a linkage pathway(s).

Agonist families can be distinguished by the fraction of their binding energy that is applied to receptor activation. Results so far suggest that there are 2 populations with regard to this fraction, at $52 \%$ and at $40 \%$ (Fig. 4). The $\alpha$ Y190A mutation shifts ACh efficiency from the higher to the lower population, raising the possibility that the distribution of efficiency values could be modal rather than continuous. If so, this 
suggests that the AChR binding pocket can adopt only a limited number of discrete $\mathrm{C}$ and $\mathrm{O}$ shapes rather than a continuum. Certainly, the efficiency of more ligands needs to be determined to test this possibility.

Knowing agonist efficiency is useful because it allows affinity to be estimated from efficacy ( $\mathrm{EC}_{50}$ from $\left.\mathrm{P}_{\mathrm{O}}{ }^{\max }\right)$. This ability is of potential clinical relevance because it can facilitate drug screening, in particular because efficiencies can estimated from structure alone. There are stipulations with regard to estimating agonist efficiency from a CRC. First, the CRC must be comprised of absolute responses rather than those normalized to the maximum. With cellular responses, normalized CRCs are far more common because it is difficult to know the number of receptors contributing to the response. Our approach (single-channels) circumvents this problem because it ensures that exactly 1 receptor contributed to $\mathrm{P}_{\mathrm{O}}$. One way to make a CRC from absolute, whole-cell responses is to count the number of receptors (Chang, Ghansah, Chen, Ye \& Weiss, 2002) or to calibrate each response to that of an agonist having a known, absolute response. Second, the CRC should be largely uncontaminated by events outside the core activation scheme (Fig. 1). Whole-cell responses arising from multiple, functionally-different receptor populations, or generated by slow agonist application onto cells bearing receptors that desensitize relatively rapidly may not produce CRCs that match Eqs. 3 and 4. For many receptors, the equations that relate CRC parameters with binding and gating constants may be more complicated than those we have used for AChRs.

Figure Legends

Figure 1. Receptor activation cycles (Scheme 1) .

Receptors adopt alternatively resting $(\mathrm{C})$ or active $(\mathrm{O})$ conformations under the influence of agonists (A). Horizontal, binding and vertical, gating. $\mathrm{E}_{\mathrm{n}}$, gating equilibrium constant with $\mathrm{n}$ bounds agonists; $\mathrm{K}_{\mathrm{dC}}$, equilibrium dissociation constant to $\mathrm{C}$ (low affinity); $\mathrm{K}_{\mathrm{dO}}$, equilibrium dissociation constant to $\mathrm{O}$ (high affinity). In adult-, wild-type human endplate AChRs with 2 bound neurotransmitter molecules, favorable binding energy generated in the $\mathrm{A}_{2} \mathrm{C}-\mathrm{A}_{2} \mathrm{O}$ transition increases $\mathrm{E}_{2}$ compared to $\mathrm{E}_{0}$ by more than 30-million fold. Scheme 2 (bold) is the main physiological activation pathway.

Figure 2. Efficiencies from CRCs .

A. The response $\left(\mathrm{P}_{\mathrm{O}}\right)$ as a function of [agonist]. Symbols, (Jadey \& Auerbach, 2012); lines, fits by Eq. 1 (Table 1). There is an inverse correlation between $\mathrm{EC}_{50}$ and $\mathrm{P}_{\mathrm{O}}{ }^{\max }$. B. Agonist efficiencies (the fraction of binding energy used for gating) calculated from $\mathrm{EC}_{50}$ and $\mathrm{P}_{\mathrm{O}}{ }^{\max }$ (Eqns. 2-5). Despite the wide ranges in $\mathrm{EC}_{50}$ and $\mathrm{P}_{\mathrm{O}}{ }^{\max }$, the agonists all have approximately the same efficiency of $53 \pm 2 \%$ (dashed line). C. Agonist structures. TMA, tetramethylammonium; ACh, acetylcholine; CCh, carbamylcholine; Ana, anabasine; Nor, nornicotine; DMP dimethylpyrrolidinium; DMT, dimethylthiazolodinium. Red, key nitrogen atom in the agonist's head group.

Figure 3. More efficiencies from CRCs .

A. $\mathrm{P}_{\mathrm{O}}$ as a function of [agonist] for Epi $(, \mathrm{n}=4)$, $\operatorname{Ebx}(, \mathrm{n}=3), \operatorname{Var}(, \mathrm{n}=2), \mathrm{Cyt}(, \mathrm{n}=4), 4 \mathrm{OH}-\mathrm{B}(, \mathrm{n}=3)$ and $3 \mathrm{OH}-\mathrm{B}(, \mathrm{n}=5) \cdot \mathrm{EC}_{50}$ and $\mathrm{P}_{\mathrm{O}}{ }^{\max }$ were estimated by Eq. 1 (Table 1). B. Agonist efficiencies calculated from fitted CRC parameters. The efficiencies of 3OH-P and 4OH-B are similar to those of the agonists shown in Fig. 1 (51\%) whereas those for Epi, Ebx, Var and Cyt smaller (40\%; dashed line). Shaded, agonist efficiencies at the $\alpha$-d binding site (Nayak, Vij, Bruhova, Shandilya \& Auerbach, 2020). C. Agonist structures. Epi, epibatidine; Ebx, epiboxidine; Var, varenicline; Cyt, cytisine; 3OH-P, 3-hydroxy-propyltrimethyammonium; 4OH-B, 4-hydroxy-butyltrimethylammonium, Atx, anatoxin-A; Aza, azabicycloheptane. Red, key nitrogen atom in the agonist's head group.

Figure 4. Agonist efficiency versus head-group volume.

Efficiencies were calculated from CRCs $(\mathrm{n}=13)$ or from single-channel kinetics $(\mathrm{n}=5)$. Crosses mark centroids of two clusters: $\eta=52 \pm 2 \%$, volume $=70 \pm 8 \mathrm{~A}^{3}$ (red) and $\eta=40 \pm 5 \%$, volume $=101 \pm 11.2 \mathrm{~A}^{3}$ (blue). Ebx from $\mathrm{CRC}$ analysis (gray) was excluded from the cluster analysis. Larger-volume agonists have lower efficiencies.

Figure 5. ACh efficiency in AChRs with a binding site mutation. 
Mutations were at $\alpha$-d and $\alpha$-e binding sites (Purohit, Bruhova, Gupta \& Auerbach, 2014): $\alpha$ Y93 (W,H,A,F, S), $\alpha \mathrm{W} 149$ (F, Y, A), $\alpha$ Y190 (F, W, A), $\alpha$ Y198 (F,H,W,S,T,L,A), $\varepsilon$ P121 (L,Y,G). The only mutation that reduced efficiency significantly was $\alpha$ Y190A.

Figure 6. Estimating affinity from efficacy.

If $\eta$ and $\mathrm{E}_{0}$ are known, $\mathrm{EC}_{50}$ can be calculated from the maximum response. A. Log-log plot of $\mathrm{EC}_{50}$ values calculated from $\mathrm{P}_{\mathrm{O}}$ max (Eq. 7) versus $\mathrm{EC}_{50}$ values estimated by fitting CRCs (Eq. 1), with linear fit (slope $=1.08, \mathrm{R}^{2}=0.85$ ). red, $\eta=52 \%$ agonists and blue, $\eta=40 \%$ agonists (Fig. 4). B. CRCs calculated from $\mathrm{P}_{\mathrm{O}}$ max (lines) superimpose approximately on experimental $\mathrm{P}_{\mathrm{O}}$ measurements (symbols).

Figure 7. Estimating intrinsic gating from a CRC.

Unliganded gating equilibrium constants $\left(\mathrm{E}_{0}\right)$ estimated from CRC parameters (Table 1). The assumed $\eta$ values were either $52 \%$ (red) or $40 \%$ (blue). The calculated $\mathrm{E}_{0}$ is approximately the same regardless of $\eta$ and close to the actual value of $4.5 \times 10^{-7}$ (dashed line).

References

Auerbach A (2012). Thinking in cycles: MWC is a good model for acetylcholine receptor-channels. J Physiol 590: 93-98.

Bruhova I, \& Auerbach A (2017). Molecular recognition at cholinergic synapses: acetylcholine versus choline. J Physiol 595:1253-1261.

Bruhova I, Gregg T, \& Auerbach A (2013). Energy for wild-type acetylcholine receptor channel gating from different choline derivatives. Biophys J 104: 565-574.

Chang Y, Ghansah E, Chen Y, Ye J, \& Weiss DS (2002). Desensitization mechanism of GABA receptors revealed by single oocyte binding and receptor function. J Neurosci 22: 7982-7990.

Colquhoun D (1998). Binding, gating, affinity and efficacy: the interpretation of structure-activity relationships for agonists and of the effects of mutating receptors. Br J Pharmacol 125: 924-947.

Elenes S, \& Auerbach A (2002). Desensitization of diliganded mouse muscle nicotinic acetylcholine receptor channels. J Physiol 541: 367-383.

Gupta S, \& Auerbach A (2011). Temperature dependence of acetylcholine receptor channels activated by different agonists. Biophys J 100: 895-903.

Jackson MB (1986). Kinetics of unliganded acetylcholine receptor channel gating. Biophys J 49: 663-672.

Jadey S, \& Auerbach A (2012). An integrated catch-and-hold mechanism activates nicotinic acetylcholine receptors. J Gen Physiol 140:17-28.

Jadey S, Purohit P, \& Auerbach A (2013). Action of nicotine and analogs on acetylcholine receptors having mutations of transmitter-binding site residue alphaG153. J Gen Physiol 141: 95-104.

Jadey SV, Purohit P, Bruhova I, Gregg TM, \& Auerbach A (2011). Design and control of acetylcholine receptor conformational change. Proc Natl Acad Sci U S A 108: 4328-4333.

Jha A, Purohit P, \& Auerbach A (2009). Energy and structure of the M2 helix in acetylcholine receptorchannel gating. Biophys J 96:4075-4084.

Karlin A (1967). On the application of "a plausible model" of allosteric proteins to the receptor for acetylcholine. J Theor Biol 16:306-320.

Lape R, Colquhoun D, \& Sivilotti LG (2008). On the nature of partial agonism in the nicotinic receptor superfamily. Nature 454:722-727. 
Monod J, Wyman J, \& Changeux JP (1965). On the Nature of Allosteric Transitions: A Plausible Model. J Mol Biol 12: 88-118.

Mukhtasimova N, Free C, \& Sine SM (2005). Initial coupling of binding to gating mediated by conserved residues in the muscle nicotinic receptor. J Gen Physiol 126: 23-39.

Mukhtasimova N, Lee WY, Wang HL, \& Sine SM (2009). Detection and trapping of intermediate states priming nicotinic receptor channel opening. Nature 459: 451-454.

Nayak TK, \& Auerbach A (2017). Cyclic activation of endplate acetylcholine receptors. Proc Natl Acad Sci U S A 114:11914-11919.

Nayak TK, Purohit PG, \& Auerbach A (2012). The intrinsic energy of the gating isomerization of a neuromuscular acetylcholine receptor channel. J Gen Physiol 139: 349-358.

Nayak TK, Vij R, Bruhova I, Shandilya J, \& Auerbach A (2020). Correction: Efficiency measures the conversion of agonist binding energy into receptor conformational change. J Gen Physiol 152.

Nicolai C, \& Sachs F (2013). Solving ion channel kinetics with the QuB software. Biophysical Reviews and Letters 8: 191-211.

Pettersen EF, Goddard TD, Huang CC, Couch GS, Greenblatt DM, Meng EC, et al. (2004). UCSF Chimera-a visualization system for exploratory research and analysis. J Comput Chem 25: 1605-1612.

Purohit P, \& Auerbach A (2009). Unliganded gating of acetylcholine receptor channels. P Natl Acad Sci USA 106: 115-120.

Purohit P, Bruhova I, Gupta S, \& Auerbach A (2014). Catch-and-Hold Activation of Muscle Acetylcholine Receptors Having Transmitter Binding Site Mutations. Biophysical Journal 107: 88-99.

Purohit P, Gupta S, Jadey S, \& Auerbach A (2013). Functional anatomy of an allosteric protein. Nat Commun 4: 2984.

Purohit Y, \& Grosman C (2006). Estimating binding affinities of the nicotinic receptor for low-efficacy ligands using mixtures of agonists and two-dimensional concentration-response relationships. J Gen Physiol 127: 719-735.

Qin F (2004). Restoration of single-channel currents using the segmental k-means method based on hidden Markov modeling. Biophys J 86:1488-1501.

Qin F, Auerbach A, \& Sachs F (1997). Maximum likelihood estimation of aggregated Markov processes. Proc Biol Sci 264: 375-383.

Sakmann B, Patlak J, \& Neher E (1980). Single acetylcholine-activated channels show burst-kinetics in presence of desensitizing concentrations of agonist. Nature 286: 71-73.

Sauguet L, Shahsavar A, Poitevin F, Huon C, Menny A, Nemecz A, et al. (2014). Crystal structures of a pentameric ligand-gated ion channel provide a mechanism for activation. Proc Natl Acad Sci U S A 111: 966-971.

Schroeder DV (2000) An introduction to thermal physics . Addison Wesley: San Francisco, CA.

Tripathy S, Zheng W, \& Auerbach A (2019). A single molecular distance predicts agonist binding energy in nicotinic receptors. J Gen Physiol 151: 452-464. 
Fig. 1

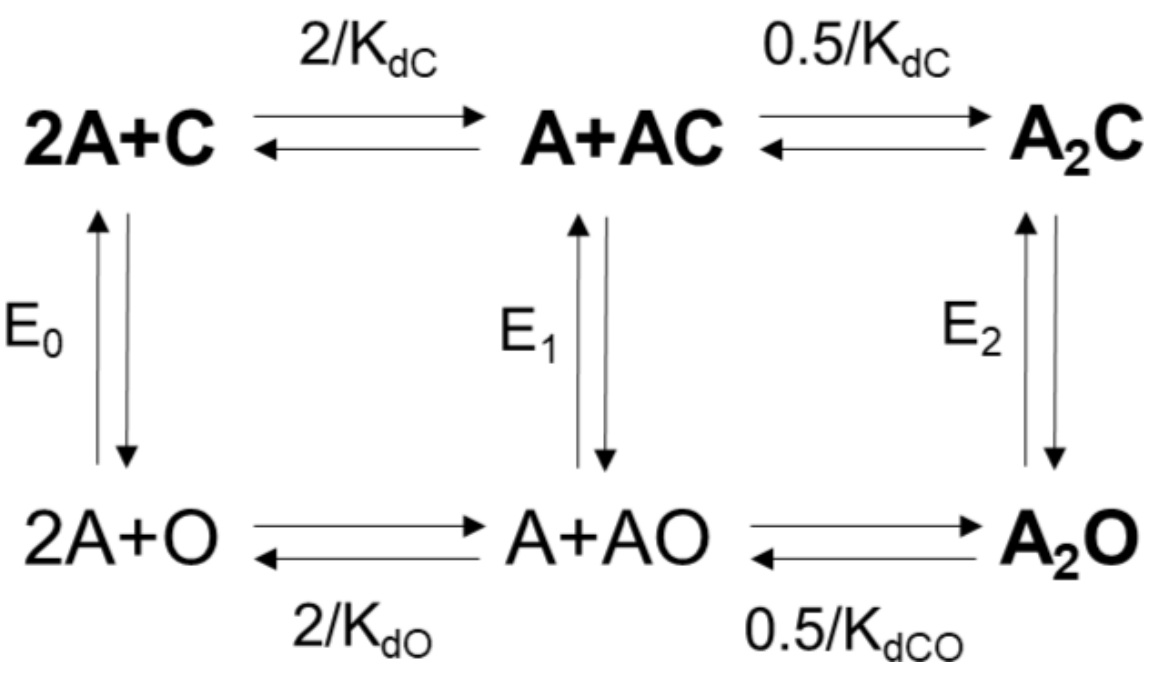

Fig. 2
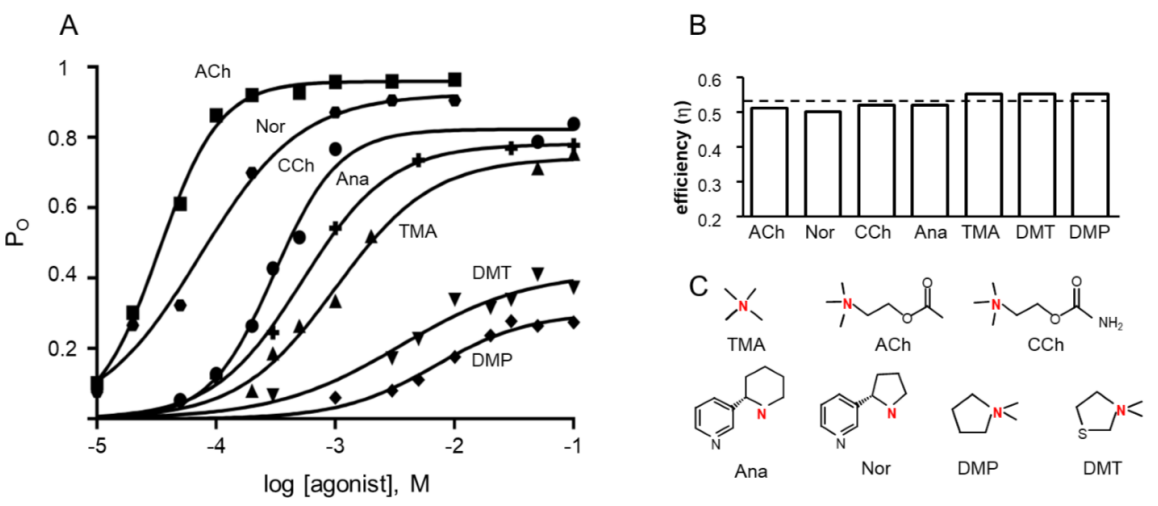
Fig. 3
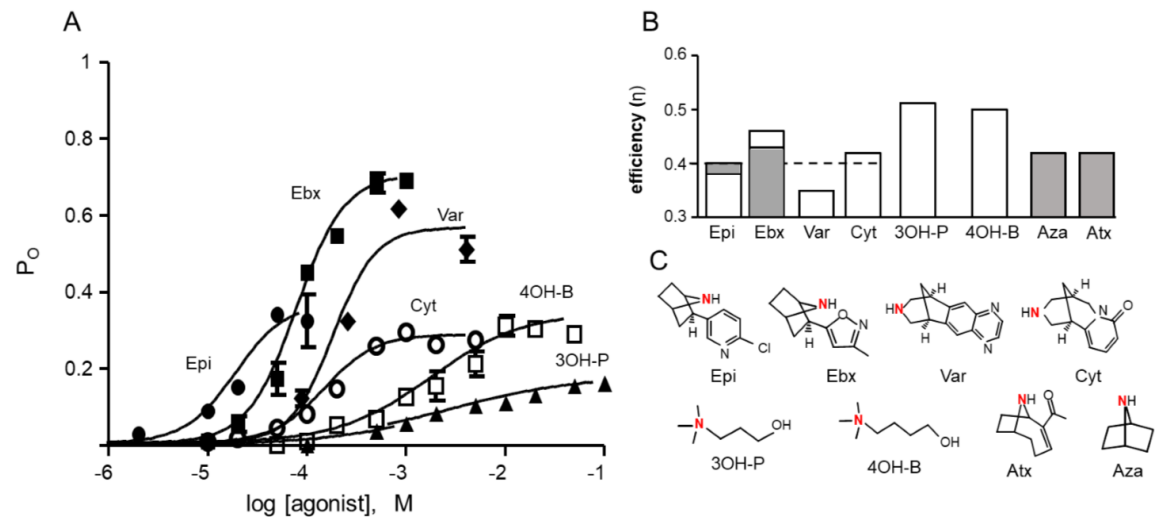

Fig. 4

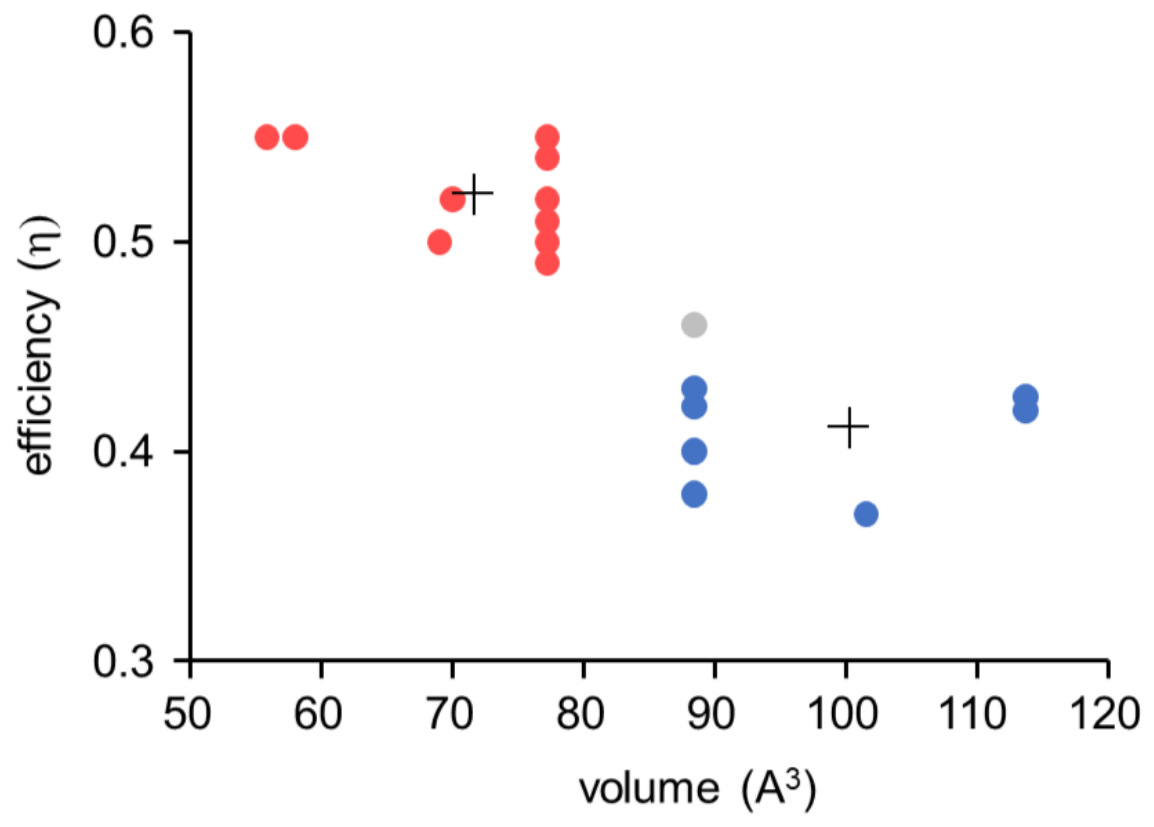


Fig. 5

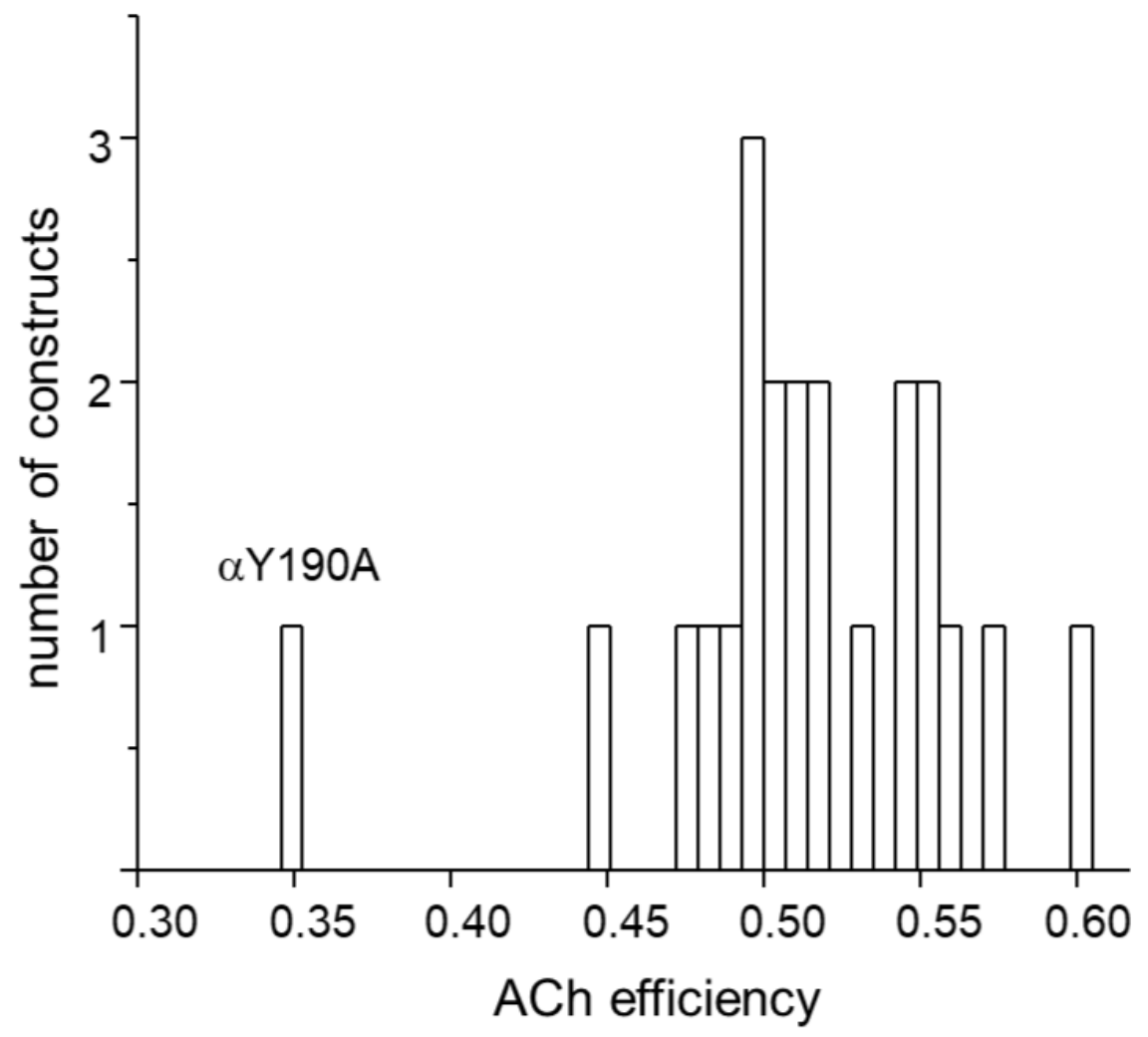

Fig. 6
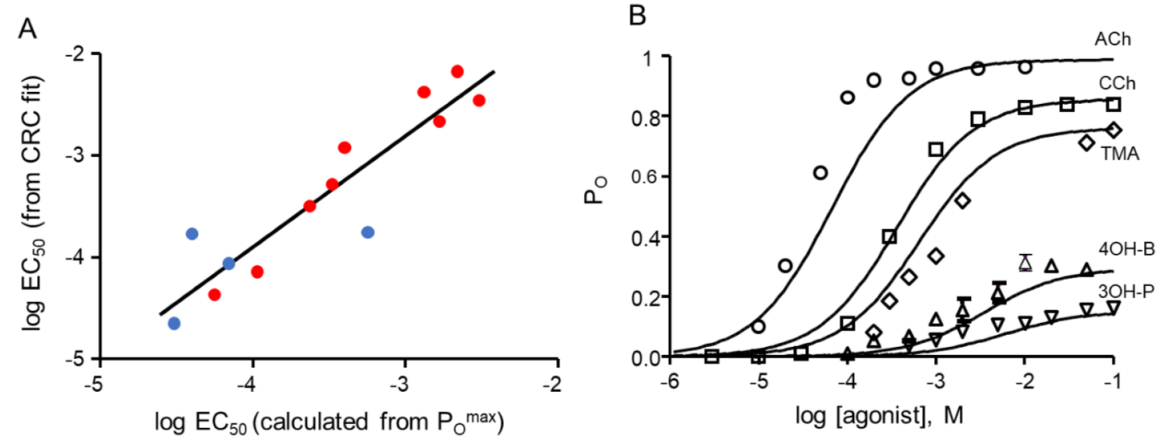
Fig. 7

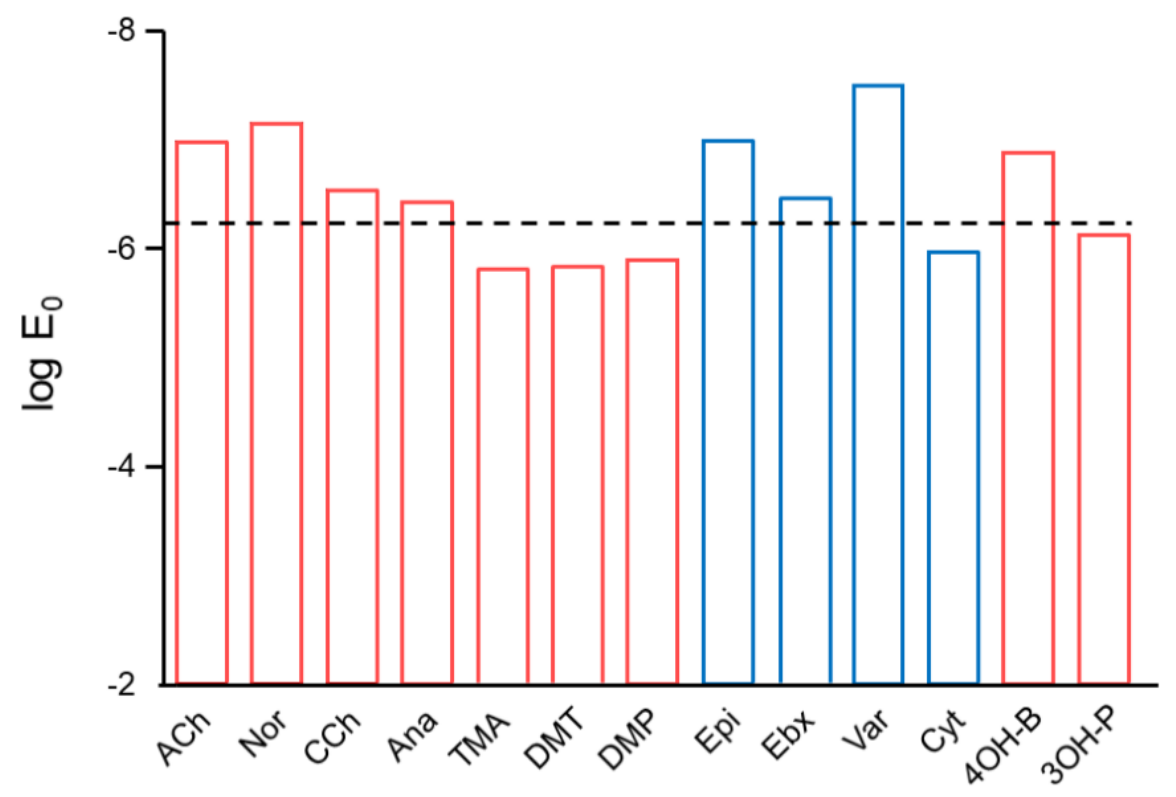

\section{Hosted file}

Table_1.docx available at https://authorea.com/users/298571/articles/427865-estimatingagonist-efficiency-from-concentration-response-curves

\section{Hosted file}

Table_2.docx available at https://authorea.com/users/298571/articles/427865-estimatingagonist-efficiency-from-concentration-response-curves 